\title{
Characteristics of Sensor Response of a Difference Interferometric Slab Optical Waveguide Refractive Index Sensor with a Prism Coupling Method
}

\author{
Kin-ichi TsunOda ${ }^{\dagger}$, Sae KIKUChI, Kiyohisa Nomura, Katsuyuki Aizawa, \\ Kanae Окамото and Hideo AKAIwA
}

Department of Chemistry, Gunma University, Kiryu 376-8515, Japan

\begin{abstract}
We developed a difference interferometric slab optical waveguide (SOWG) refractive index (RI) sensor for flow analysis which used a prism coupling method. The highest sensitivity of $7.2 \times 10^{-7}$ ( $\Delta$ RI unit) was obtained with a glass SOWG fabricated with a radio frequency sputtering method for solution samples such as sucrose solutions and mixtures of ethanol and water; here sensitivity was defined as the $\Delta \mathrm{RI}$ which gives the signal intensity of $10^{-4}$ in absorbance unit. The sensitivity is almost the same level as that of a surface plasmon resonance sensor.
\end{abstract}

Recently various kinds of planer optical waveguides have been applied to integrated opto-chemical ${ }^{1-4}$ and -bio sensors ${ }^{5-7}$, in which the light absorption or the refractive index (RI) change due to analytes on the sensor surface is monitored. We have also been applying various types of glass slab optical waveguides (SOWG) to an absorption detector to detect trace amounts of several dyes, iron(II) or silicic acid. ${ }^{8-11}$

As for an RI sensor using a planer optical waveguide, several types of so-called "leakage mode sensors" have been developed. In such sensors, the RI change at a coupler of the waveguide with source light, a grating coupler $^{5}$ or a "resonance mirror" type coupler ${ }^{6}$, causes the changes of the coupling efficiency and/or the coupling conditions, e.g., the angle of the out-coupled light beam and so on; these changes have been utilized for chemical sensing. However, it is difficult to fabricate such sensing elements in an ordinary chemical laboratory. On the other hand, when an ordinary SOWG system with a prism coupler or an end coupler is used in a waveguide chemical sensor, a sample does not touch with the coupler directly; the coupling conditions are presumed not to change due to the kinds of the samples. Hence, another idea has to be introduced in order to construct an RI sensor using an SOWG with such a coupler. One of the approaches is to employ a MachZehnder interferometer with a planer optical waveguide. Although several works have been reported on such type of sensors ${ }^{4,7}$, special microfabrication techniques should be necessary for making the interferometer; moreover, the optical configuration is rather complicated. Chen et al. have proposed a RI sensor using a glass SOWG having a tapered velocity coupler. ${ }^{12}$ Although the sensor should be classified as a leakage mode sensor in principle, any kind of coupling methods

$\doteqdot$ To whom correspondence should be addressed. can be used for the sensor, so, it can be a more convenient sensor system. Tentatively, it still lacks sufficient sensitivity $\left(\sim 10^{-4} \Delta \mathrm{RI}\right.$ unit). We have also proposed an RI SOWG sensor with a prism coupler using an optical position sensor. ${ }^{13}$ However, the mechanism of its response has not been clearly understood yet.

Recently, a difference interferometric RI sensor has also been proposed; this is based on the differential phase modulation between the two orthogonal polarization modes of guided lights. ${ }^{14-19}$ Although this type of RI sensor is relatively simple in optical configuration and is resistant to external disturbances such as temperature and/or mechanical fluctuation, it had been believed that only an end coupling method can be utilized with this sensor to couple source light with an SOWG, because the two polarized guiding modes, i.e., transverse electric (TE) mode and transverse magnetic (TM) mode, have to be excited at the same time with almost the same efficiency. ${ }^{19}$ However, the end coupling method is also difficult to use in an ordinary chemical laboratory because it requires the fine adjustment of optical configuration.

In this study, we have found that highly sensitive difference interferometric response can also be obtained even with an SOWG using a prism coupling method, which is the easiest to use for chemists. The potentiality of this sensor as an RI detector for a flow analysis system is evaluated by comparing with a surface plasmon resonance (SPR) sensor.

\section{Theory}

Although the principle of the sensor response has already been described in the literature ${ }^{14-17}$, a brief description is also given here. Figure 1a shows the schematic diagram of the present SOWG sensor sys- 
a)

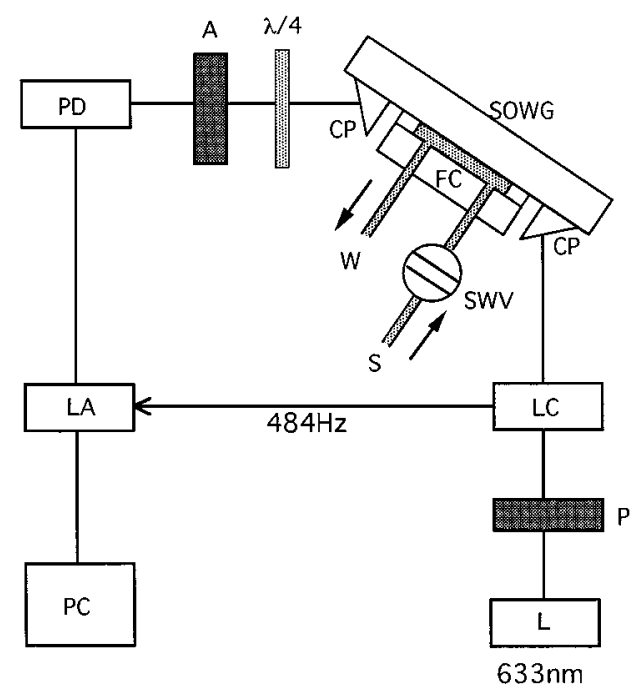

b)

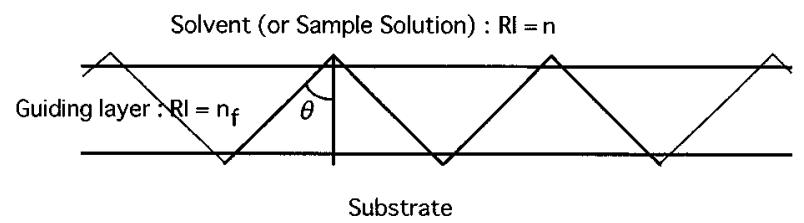

C)

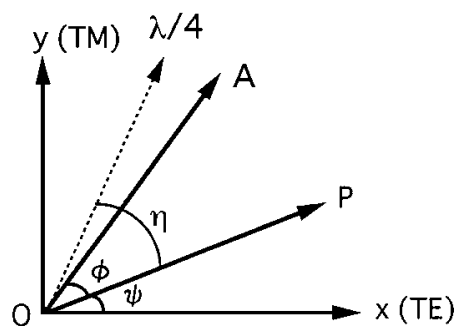

Fig. 1 Concept of the SOWG RI sensor system. a) Arrangement of the SOWG RI sensor: L, He-Ne laser; P, polarizer P; LC, light chopper $(484 \mathrm{~Hz})$; $\mathrm{CP}$, coupler prism; SOWG, slab optical waveguide; $\lambda / 4, \lambda / 4$ phase plate; A, polarizer A; PD, photo-diode; LA, lock-in amplifier; PC, personal computer; FC, flow cell; SWV, six way valve; S, solvent reservoir; W, sample waste. b) The imaginary light path in the SOWG sensor: $\theta$, total internal reflection angle. c) The angles of polarizers $\mathrm{P}$ and $\mathrm{A}$ and a $\lambda / 4$ phase plate on the coordinates of the orthogonal polarization modes TE and TM.

tem. The SOWG is placed between two polarizers, $\mathrm{P}$ and $\mathrm{A}$. Monochromatic light polarized by the polarizer $\mathrm{P}$ is applied to the SOWG with a prism coupler; the guided light passing through the other polarizer $\mathrm{A}$ is detected with a photodiode. In Fig. 1b, the structure of the SOWG sensor and the imaginary light path in the SOWG are also shown. The SOWG directly touches a solution sample and the small change in RI of the solu- tion is to be detected. Moreover, the angles of the polarizers $\mathrm{P}$ and $\mathrm{A}$ are expressed on the co-ordinates of the orthogonal polarization modes TE and TM in Fig. 1c. The response of this sensor is based on the apparent anisotropic nature of the SOWG between TE and TM modes.

Change in phase difference between TE and TM modes caused by changes in RI of solution

When the SOWG is used as an absorption detector for liquid samples, $L_{\text {eff }}(\mathrm{cm})$ is defined according to Lambert-Beer's law as

$$
A_{\mathrm{SOWG}}=\varepsilon C L_{\mathrm{eff}}
$$

where $A_{\text {Sowg }}$ is an absorbance obtained with the SOWG detector, $\varepsilon$ a molar absorptivity of the analyte $\left(\mathrm{dm}^{3}\right.$ $\mathrm{mol}^{-1} \mathrm{~cm}^{-1}$ ) and $c$ a concentration of an analyte in a liquid sample $\left(\mathrm{mol} \mathrm{dm}^{-3}\right)$. According to a textbook of evanescent spectroscopy ${ }^{20}$, the $L_{\mathrm{eff}}{ }^{\mathrm{TM}}$ is known to be about 1.2 to two times greater than the $L_{\mathrm{eff}}^{\mathrm{TE}}$. The degree of the difference between the two effective path lengths $\left(\Delta L_{\text {eff }}=L_{\text {eff }}{ }^{\mathrm{TM}}-L_{\text {eff }}{ }^{\mathrm{TE}}\right)$ depends on the RI parameters of the SOWG. The difference $\left(\Delta L_{\text {eff }}\right)$ causes the phase shift $(D)$ between TE and TM modes, and the dependence of the change in the phase shift $(\Delta D)$ upon the change in the RI of a sample solution $(\Delta n)$ (Fig. 1b) is described by

$$
\Delta D=-k_{0} \Delta L_{\text {eff }} \Delta n
$$

where $k_{0}=2 \pi / \lambda_{0}, \lambda_{\mathrm{o}}$ being the wavelength in vacuo. That is, the change of the phase shift $(\Delta D)$ is proportional to $\Delta n$ and $\Delta L_{\text {eff. }}$

\section{Absorbance response of the detector to $\Delta D$}

Although the $\Delta D$ is usually detected using an analyzer system, we monitored it as an absorbance signal mainly because its experimental simplicity. When the angles $\left({ }^{\circ}\right.$ ) of $\mathrm{P}$ and $\mathrm{A}$ are set at 45 and 0 in Fig. $1 \mathrm{c}$, respectively, and $\Delta D$ is small enough, the output signal in the absorbance unit is described by

$$
A=\frac{\sin D_{0}}{2.303\left(1+\sin D_{0}\right)} k_{0} \Delta L_{\text {eff }} \Delta n
$$

where $D_{0}$ is a baseline value for $D$, that is, the $D$ value when solvent (carrier solution) is introduced into the sensor. The $\Delta D$ value is the change of the $D$ value from the baseline due to sample introduction. From Eq. (3), it is apparent that the response of the sensor is influenced greatly by the $D_{0}$ value, although the $D_{0}$ value is adjusted by inserting a $\lambda / 4$ phase plate anywhere between the two polarizers $\mathrm{P}$ and A. Moreover, it should be noted that the linearity of the response is not obtained except for that the range of that $\Delta D$ is small enough, when the absorbance detection is applied. 


\section{Experimental}

\section{Slab optical waveguide}

Three kinds of slab optical waveguide (SOWG) as shown in Fig. 2 were used in this study. A potassium ion doped glass SOWG (K+-SOWG, Fig. 2a) was fabricated by an ion-exchange process on commercial sodalime slide glasses (S-1214 from Matsunami Glass Inc., Japan) in molten potassium nitrate at $673 \mathrm{~K}$ for 30 min. ${ }^{8,9}$ The second one is a glass SOWG having a tapered velocity coupler (SP-SOWG, Fig. 2b), which was originally proposed by Itoh and Madou to enhance the sensitivity of the SOWG measurements. ${ }^{21} \mathrm{~A} \mathrm{~K}^{+}$ SOWG was fabricated first by an ion-exchange process on a borosilicate glass slide $\left(n_{\mathrm{D}}=1.474,25 \times 70 \mathrm{~mm}\right)$ in molten $\mathrm{KNO}_{3}$ at $723 \mathrm{~K}$ for $90 \mathrm{~min}$. Then, a thin film (ca. $1.5 \mu \mathrm{m}$ thick, $10 \mathrm{~mm}$ wide) of Corning 7059 glass, which had tapered slopes at both ends, was deposited onto the center of the $\mathrm{K}^{+}-\mathrm{SOWG}$ with a custom-made radio frequency (rf)-sputtering apparatus (Veetech, Japan). Sputtering conditions were the same as those of the previous paper. ${ }^{10}$ These two SOWGs supported only $n=0$ mode of guided light, that is, $\mathrm{TE}_{0}$ and $\mathrm{TM}_{0}$ modes. On the other hand, the third one was a multimode sheet glass SOWG (SG-SOWG, Fig. 2c) assembled according to our previous paper, in which the guiding layer was made of thin sheet glass $(50 \mu \mathrm{m}$ thick, quartz glass, $\left.n_{\mathrm{D}}=1.459\right)$ and the clad was the air. ${ }^{22}$

\section{Measurement system}

As is shown in Fig. 1a, a He-Ne laser (632.8 nm, 2 $\mathrm{mW}$, random polarization) was used for a light source. The SOWG was mounted on a $360^{\circ}$ rotational stage with X-Y-Z translation. The laser light was modulated by a light chopper at $487 \mathrm{~Hz}$ and was coupled into the SOWG with a prism coupler $\left(n_{\mathrm{D}}=1.8785\right)$ and the guided light was out-coupled with another prism coupler. Polarizers $\mathrm{P}$ and $\mathrm{A}$ were placed before and after the SOWG, respectively. The $\lambda / 4$ phase plate was inserted between the SOWG and the polarizer A if indicated. The polarizers were rotated manually to adjust the $\psi$ and $\phi$ values in Fig. 1c. The guided light was detected with a photodiode and the signal was amplified with a 5600A digital lock-in amplifier (NF Circuit Block, Japan) and fed into a personal computer through a General Purpose Interface Bus (GP-IB). The flow cell was placed on the SOWG with a polytetrafluoroethylene (PTFE) block and a PTFE spacer (0.5 mm thick). The physical cell length was $1.0 \mathrm{~cm}$, and the cell volume was ca. $15 \mathrm{~mm}^{3}$. Deionized water as a carrier was pumped with an SNK DMX-2300T double plunger pump (Sanuki Industry, Japan) at $0.6 \mathrm{~cm}^{3} \mathrm{~min}^{-1}$. A sample solution $\left(0.5 \mathrm{~cm}^{3}\right)$ was introduced into the carrier stream via a Rheodyne 5020 loop injector (Cotati, USA). Mixtures of ethanol and distilled water and various concentrations of sucrose solutions were used as RI standards. Moreover, dilute Methylene Blue (MB)

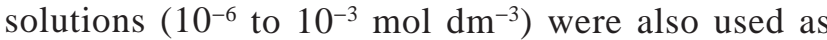

a)

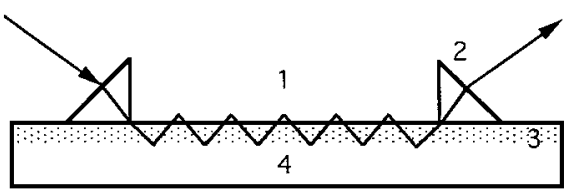

b)

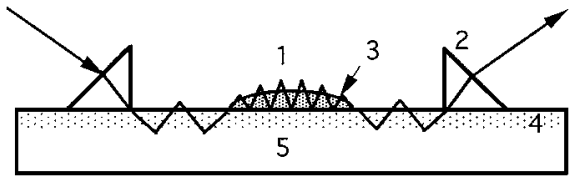

c)

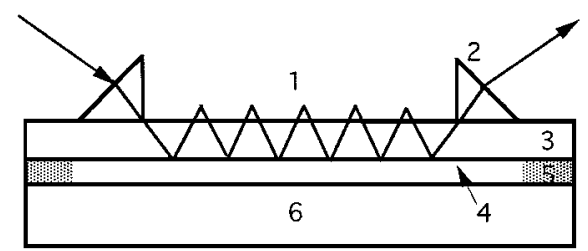

Fig. 2 Slab optical waveguides (SOWGs). a) Potassium ion doped glass SOWG $\left(\mathrm{K}^{+}-\mathrm{SOWG}\right)$ : 1 , water $\left(n_{\mathrm{D}}=1.333\right)$; 2 , prism; 3, $\mathrm{K}^{+}$doped layer; 4, substrate (borosilicate glass, $\left(n_{\mathrm{D}}=1.472\right)$. b) Glass SOWG fabricated with a sputtering method (SP-SOWG): 1, water $(n=1.333) ; 2$, prism; 3, sputtered layer (Corning 7059 glass, $n=1.530$ ); $4, \mathrm{~K}^{+}$doped layer; 5 , substrate (borosilicate glass, $n=1.472$ ). c) Multi-mode sheet glass SOWG (SG-SOWG): 1, water $(n=1.333) ; 2$, prism; 3, sheet glass (50 $\mu \mathrm{m}$ thick, Corning 0211, $n=1.523$ ); 4, clad (the air, $n=1.000)$; 5, FEP film (12 $\mu$ m thick); 6 , substrate (borosilicate glass).

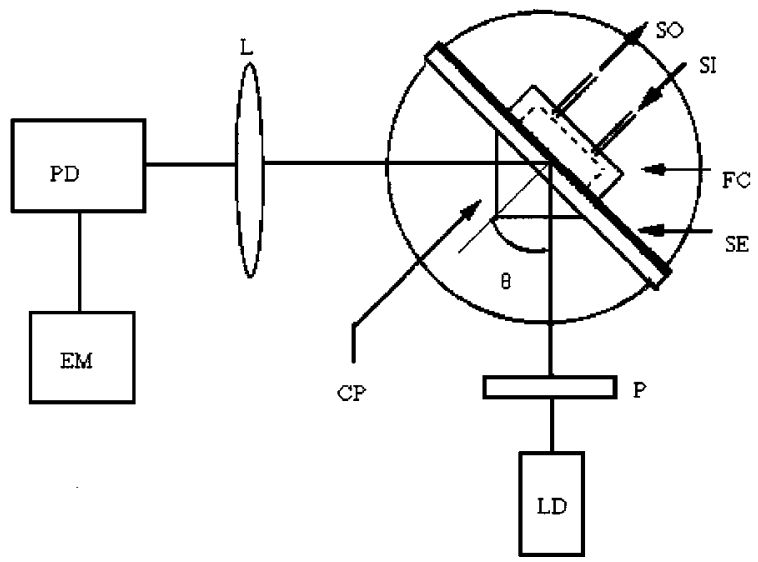

Fig. 3 Schematic diagram of the SPR sensor system. LD, laser diode $(670 \mathrm{~nm}$ or $780 \mathrm{~nm})$; P, polarizer; $\mathrm{CP}$, coupler prism $\left(n_{\mathrm{D}}=1.805\right)$; SE, sensing element with a $\mathrm{Ag}$ thin layer; L, lens; PD, photodiode; EM, electrometer.

sample solutions.

\section{Experimental measurement of $\Delta L_{\text {eff }}$}

Bromothymol Blue (BTB) solutions (in $10 \mathrm{mmol} \mathrm{dm}^{-3}$ phosphate buffer ( $\mathrm{pH}$ 8.0)) were used as absorbance standards to measure the $\Delta L_{\text {eff }}$ of the SOWG, because BTB molecules are not adsorbed on a glass surface. The polarizer $\mathrm{P}$ was removed and the polarizer $\mathrm{A}$ was set to pass the guided light of only TE or TM mode. The 

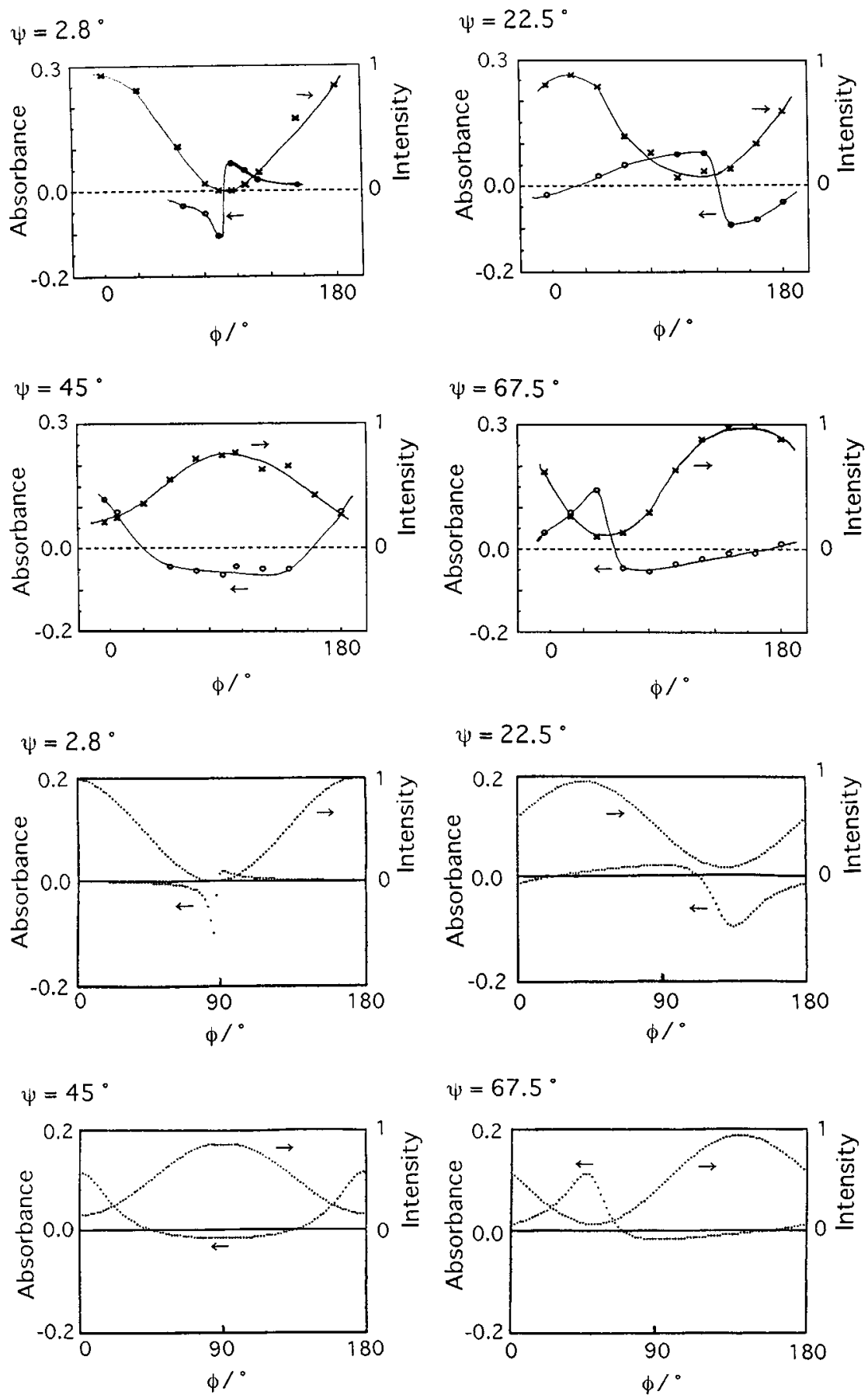

Fig. 4 Dependencies of the absorbance response and transmitted light intensity upon the angles of polarizers $\mathrm{P}(\psi)$ and $\mathrm{A}(\phi)$. a) Experiment: sample, sucrose $10 \%$ solution; SOWG, $\mathrm{K}^{+}-\mathrm{SOWG}$. b) Simulation: $\Delta D=10^{\circ}$. A certain amount of stray light (10\% of incident light) was taken into calculation. Moreover, the $D_{0}$ value was postulated as $120^{\circ}$.

absorbance due to the BTB solution was measured with the SOWG detector. $\Delta L_{\text {eff }}$ values for TE and TM modes $(\mu \mathrm{m})$ were calculated from the following equation:

$$
L_{\text {eff }}=\frac{A_{\text {sowG }}}{A_{\text {lcm }}} \times 10^{4}
$$

where $A_{\text {SowG }}$ is the absorbance obtained by the SOWG detector and $A_{1 \mathrm{~cm}}$ is that obtained by a conventional spectrophotometer (cell length $\left.=1 \mathrm{~cm}\left(10^{4} \mu \mathrm{m}\right)\right)$. Then, the $\Delta L_{\text {eff }}\left(=L_{\text {eff }}{ }^{\mathrm{TM}}-L_{\text {eff }}{ }^{\mathrm{TE}}\right)$ was calculated.

\section{Surface plasmon resonance sensor measurements}

Figure 3 shows the experimental setup of surface plasmon resonance (SPR) measurements for the RI change of solution samples. The sensing element is a slide glass (S-1214 from Matsunami Glass Inc., Japan) on which a thin silver layer (ca.70 nm thick) was 


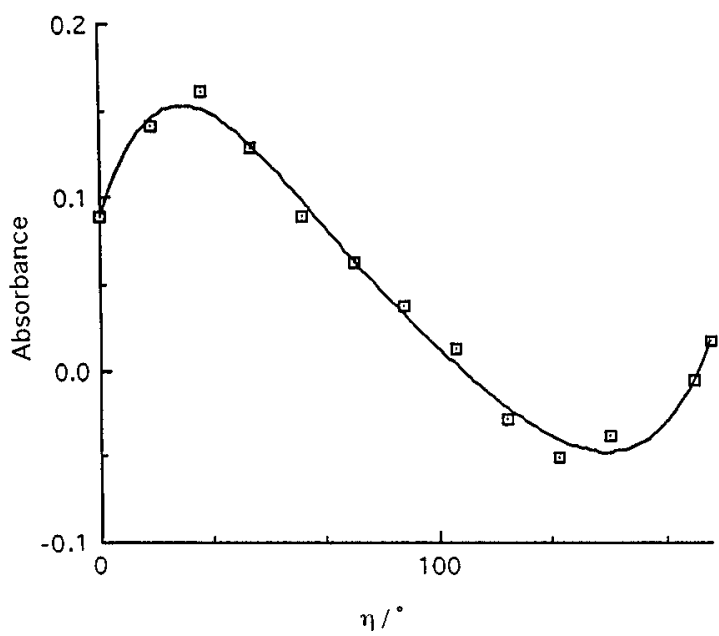

Fig. 5 Dependence of the absorbance response upon the angle of $\lambda / 4$ phase plate. Sample, $10 \%$ sucrose solution; SOWG, $\mathrm{K}^{+}$-SOWG; angles of polarizers $\mathrm{P}(\psi)$ and $\mathrm{A}(\phi): \psi=45^{\circ}$, $\phi=0^{\circ}$.

deposited with the rf-sputtering apparatus. The sensing element and the source light were coupled with a high RI coupler prism $\left(n_{\mathrm{D}}=1.805\right)$, and the out-coupled light was monitored with a photodiode which was placed where the intensity becomes the average of the intensities of the non-resonant position and the maximum resonant position. Mixtures of ethanol and distilled water and various concentrations of sucrose solutions were also used as RI standards.

\section{Results and Discussion}

Sensor response to refractive index change of solution

The dependence of transmitted light intensity $(I)$ and the absorbance response of the SOWG sensor to $10 \%$ sucrose solution upon the angles of the polarizers $\mathrm{P}$ and A was first investigated using the $\mathrm{K}^{+}$-SOWG. The results were compared with those of a computer simulation. In this experiment, a $\lambda / 4$ phase plate was not used. Figures $4 \mathrm{a}$ and $4 \mathrm{~b}$ show the results of the actual experiments and the computer simulation, respectively. As shown in these figures, the experimental results correspond very well to those of the computer simulation where the $D_{0}$ value (in Eq. (3)) was set at $D_{0}=60^{\circ}$. These results clearly show that the sensor response is based on the proposed mechanism. As shown in figures, the most stable response was obtained when the $\psi$ and $\phi$ were set at $45^{\circ}$ and $0^{\circ}$, respectively. Then, the $\lambda / 4$ phase plate was inserted and the dependence of the absorbance response on the angle of its axis was investigated at $\psi=45^{\circ}$ and $\phi=0^{\circ}$. Figure 5 show the experimental result, which corresponds well to the theoretical consideration.

Excitation of TM and TE modes with a prism coupler

As mentioned previously, the two polarized guiding a)

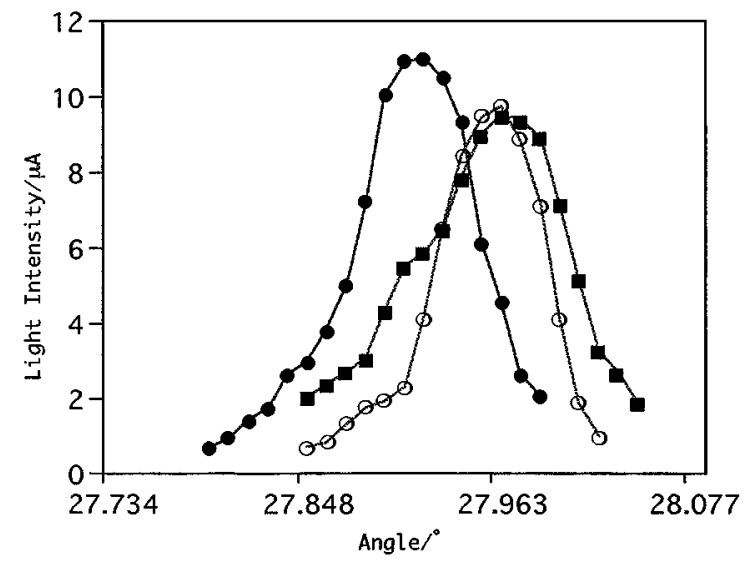

b)

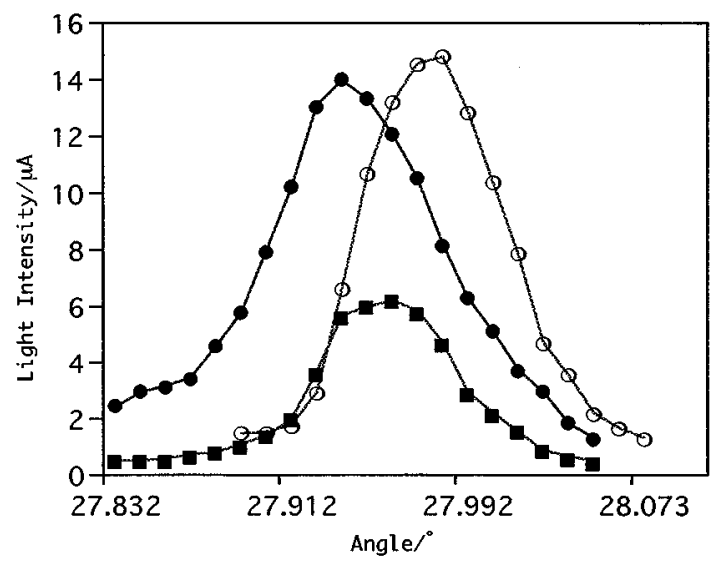

Fig. 6 Excitation of $\mathrm{TE}_{0}$ and $\mathrm{TM}_{0}$ modes with a prism coupler. The detector position (the angle $\varphi$ ) was changed with a $x$-direction-stage (Fig. 1a), and the intensities of the guided light were plotted against the angle $\varphi$. a) The incident angle of the source light was set at the optimum angle for exciting $\mathrm{TM}_{0}$ mode: $\bullet, \mathrm{TM}_{0}$ mode (angles of polarizers $\mathrm{P}(\psi)$ and $\mathrm{A}$ $\left.(\phi), \psi=90^{\circ}, \phi=0^{\circ}\right) ; 0, \mathrm{TE}_{0}$ mode (angles of polarizers $\mathrm{P}(\psi)$ and $\left.\mathrm{A}(\phi), \psi=0^{\circ}, \phi=0^{\circ}\right)$; $\mathbf{\square}$, the measurement condition of $\Delta$ RI (angles of polarizers $\mathrm{P}(\psi)$ and $\mathrm{A}(\phi): \psi=45^{\circ}, \phi=0^{\circ}$ ). b) The incident angle of the source light was set at the average of the optimum angles for exciting $\mathrm{TE}_{0}$ and $\mathrm{TM}_{0}$ modes, respectively. The symbols in the figure are the same as those of Fig. 6a).

modes, i.e., TE mode and TM mode, have to be excited at the same time with almost the same efficiency to obtain a sensitive difference interferometric response. Thus, the efficiency of the excitation of the two modes with a prism coupler was investigated for a single mode $\mathrm{K}^{+}$-SOWG. Figures $6 \mathrm{a}$ and $6 \mathrm{~b}$ show the example of the experimental results on the excitation of $\mathrm{TE}_{0}$ and $\mathrm{TM}_{0}$ modes of the SOWG, when randomly polarized $\mathrm{He}-\mathrm{Ne}$ laser light was used as the source light. The optimum incident angles of the source light to excite the two modes were slightly different from each other, as predicted from the theory of the waveguide. ${ }^{1,2,9}$ As is shown in Fig. 6a, however, the two modes were excited at the same time, even when the incident angle of the 
Table 1 The effective-path-length difference $\left(\Delta L_{\text {eff }}\right)$ and sensitivity ${ }^{\mathrm{a}}$ of SOWG RI sensor

\begin{tabular}{ccccc}
\hline SOWG & $\begin{array}{c}\Delta L_{\text {eff }}{ }^{\mathrm{b}} / \\
\mu \mathrm{m}\end{array}$ & $\begin{array}{c}\Delta L_{\text {eff }}{ }^{\mathrm{c}} / \\
\mu \mathrm{m}\end{array}$ & $\begin{array}{c}\text { Sensitivity }^{\mathrm{d}} \\
(\text { Calc. })\end{array}$ & $\begin{array}{c}\text { Sensitivity }^{\mathrm{e}} \\
(\text { Expr. })\end{array}$ \\
\hline $\mathrm{K}^{+}-$SOWG $^{\mathrm{f}}$ & - & 1 & $3.8 \times 10^{-6}$ & $1.0 \times 10^{-5}$ \\
SP-SOWG $^{\mathrm{g}}$ & - & 38 & $1.0 \times 10^{-7}$ & $7.2 \times 10^{-7}$ \\
SG-SOWG $^{\mathrm{h}}$ & & & & \\
$72^{\mathrm{i}}$ & 3.9 & 2.5 & $1.5 \times 10^{-6}$ & $6.8 \times 10^{-6}$ \\
64 & 13 & 7.7 & $7.9 \times 10^{-7}$ & $2.6 \times 10^{-6}$ \\
62 & 34 & 31 & $1.2 \times 10^{-7}$ & $1.8 \times 10^{-6}$ \\
\hline
\end{tabular}

a. The sensitivity was defined as the $\Delta n$ value (RI unit) which gives absorbance change of $10^{-4}$. Sucrose solutions were used as RI standards.

b. Calculated values. ${ }^{21}$

c. The values obtained with using the BTB standard solutions (see

Experimental section).

d. A certain amount of stray light (3\% of the incident light) was taken into calculation.

e. $\psi=45^{\circ}, \phi=0^{\circ}$.

f. A potassium ion doped glass SOWG.

g. A glass SOWG fabricated with a sputtering method.

h. A multi-mode sheet glass SOWG.

i. Internal reflection angle of guiding light ( $\theta$ in Fig. 1b)). Critical angle $=61.1^{\circ}$.

source light was set at the optimum angle for exciting the $\mathrm{TM}_{0}$ mode. A similar result was also obtained at the optimum angle for exciting the $\mathrm{TE}_{0}$ mode. Moreover, when the incident angle was set at the middle of the two angles, the $\mathrm{TE}_{0}$ and $\mathrm{TM}_{0}$ modes were excited almost equally, as shown in Fig. 6b. From these results, it was confirmed that the two modes could easily be excited at the same time even with a prism coupler.

\section{Sensor response to RI standard solutions}

As can be derived from Eq. (2), the sensitivity of the sensor is expected to depend on the $\Delta L_{\text {eff }}$ value of the SOWG sensor. Thus, various types of SOWGs of different $\Delta L_{\text {eff }}$ values were tested and their sensor responses were compared with one another. Table 1 summarizes the sensitivities of the SOWG RI sensors as well as the $\Delta L_{\text {eff }}$ values of various SOWGs tested in this study. The sensitivity was defined as the $\Delta n$ value (RI unit) which gives an absorbance change of $10^{-4}$. As shown in the table, the sensitivity of the SOWG RI sensor increased along with the increase in the $\Delta L_{\text {eff }}$ value. The highest sensitivity $\left(7.2 \times 10^{-7}\right.$ in $\Delta \mathrm{RI}$ unit) was obtained with the SP-SOWG. Moreover, the SGSOWG could also give relatively high sensitivity, considering that the guiding layer of the SG-SOWG is estimated to be about 50 times thicker than those of the $\mathrm{K}^{+}$-SOWG and SP-SOWG. This is attributable to the fact that even the light for the internal reflection angle $(\theta)$ close to the critical angle $\left(61.6^{\circ}\right.$ in this case) can propagate through the SG-SOWG as it is a multi-mode waveguide, and such a higher mode gives a relatively large $\Delta L_{\text {eff }}$ value as shown in Table 1 . The theoretical
Table 2 Sensitivity ${ }^{\mathrm{a}}$ of SPR sensor

\begin{tabular}{lc}
\hline \multicolumn{1}{c}{ Sample } & Sensitivity $^{\mathrm{a}}$ \\
\hline Mixture of ethanol and water & $3.7 \times 10^{-6}$ \\
Sucrose solution & $3.5 \times 10^{-6}$ \\
\hline
\end{tabular}

a. The sensitivity was defined as the $\Delta n$ value (RI units) which gives absorbance change of $10^{-4}$.

sensitivity of the RI sensors was also calculated where a certain amount of stray light (3\% of the incident light to SOWGs) was postulated. As shown in the table, each experimental value is almost ten-times lower than the calculated one. One of the reasons might be that the sensitivity is dependent upon the percentage of the stray light which may be caused due to loss at the coupling of the source light and the SOWG; 10 to $20 \%$ of the stray light in the incident light could roughly explain the experimental results.

The sensitivity of the SOWG RI sensor was compared with the SPR sensor. Table 2 shows the sensitivity of the SPR sensor obtained for the mixture of ethanol and distilled water and the sucrose solution. As shown in Tables 1 and 2, the sensitivities of these two sensors were at almost the same level. Moreover, the sensitivity of the present SOWG RI sensor was compared with those of the difference interferometric planer optical waveguide sensors with an end coupler. Fattinger et al. reported the sensitivity of their sensors as $5 \times 10^{-6} \Delta \mathrm{RI}$ unit. ${ }^{15}$ Lukosz et al., also reported that theirs was $2 \times 10^{-5} \Delta$ RI unit, although they insisted that the sensitivity of $2 \times 10^{-7}$ to $2 \times 10^{-8}$ ( $\Delta$ RI unit) could theoretically be expected for it. ${ }^{18}$ Although strict comparison between the present sensor and those ones are impossible, all sensitivities are comparable with one another.

Figure 7 shows the signal profiles of the sensor with the SP-SOWG which has the greatest $\Delta L_{\text {eff }}$ value (see Table 1). Although the linear response was obtained in the range for the lower concentration of the sucrose standards, so-called roll-over phenomenon was observed in the higher concentration range. This rollover phenomenon can be explained, when the $D_{0}$ value in Eq. (3) is estimated as $c a .110^{\circ}$. Although this problem limits the calibration range of the sensor, it can easily be overcome by introducing the phase analyzing system for the detection; this is now under investigation.

\section{Sensor response to Methylene Blue solution}

The Methylene Blue (MB) molecule is known to be adsorbed from its dilute aqueous solution onto glass and metal surfaces to form monolayers of the molecule. The adsorption behavior of MB can be observed by the SOWG method with absorption measurements ${ }^{9}$ and an SPR sensor. ${ }^{23}$ Although the RI change due to a dilute MB solution itself should be too small to be detected, the sensor may respond to the RI change at the SOWG surface caused by the adsorbed MB molecules. Thus, the present SOWG RI sensor was applied to the detec- 


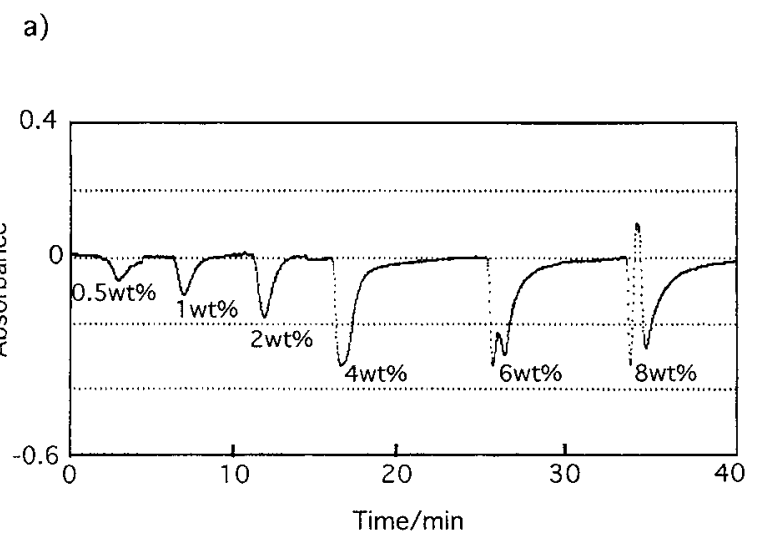

Fig. 7 Signal profiles of SOWG RI sensor. Sample, sucrose solution; SOWG, SP-SOWG; angles of polarizers P $(\psi)$ and A $(\phi) ; \psi=45^{\circ}, \phi=0^{\circ}$.

tion of the MB adsorption. However, no sensor response could be obtained even with the SP-SOWG. On the other hand, the SPR sensor showed an obvious sensor response to the dilute $\mathrm{MB}$ solution as shown in Fig. 8. These results mean that the present SOWG RI sensor is relatively insensitive to the surface phenomena, although it is sensitive to the RI change of the solution neighboring to the surface. Hence, the sensing layer thicker than $c a .100 \mathrm{~nm}$ may be necessary, when the present SOWG RI sensor is applied to the active sensing system. These characteristics of the sensor response can be explained by the intensity of the electromagnetic field at the sensor surface, which is weaker in the SOWG than in the SPR element. ${ }^{19}$

A sensitive SOWG RI sensor with ellipsometric measurements is developed for flow analysis. This system requires very simple optical configuration, and is applicable to any kind of planer type waveguide sensors. Since an evanescent wave is used for sensing, only interfacial phenomena, i.e., those in the region up to about one hundred $\mathrm{nm}$ from the surface (depending on the wavelength of source light used), can be monitored. Moreover, both light absorption and the RI change can be monitored at the same time with minor improvement in the signal treatments. Thus, this system may be useful for constructing new types of optochemical- and bio-sensors as well as for studying various interfacial phenomena.

This work was supported in part by a Grant-in-Aid for Scientific Research (No. 07640798 (C)(2)) from the Ministry of Education, Science, Sports and Culture, Japan.

\section{References}

1. M. D. Degrandpre, L. W. Burgess, P. L. White and D. S. Goldman, Anal. Chem., 62, 2012 (1990).

2. S. S. Saavedra and W. M. Reichert, Anal. Chem., 62, 2251 (1990).

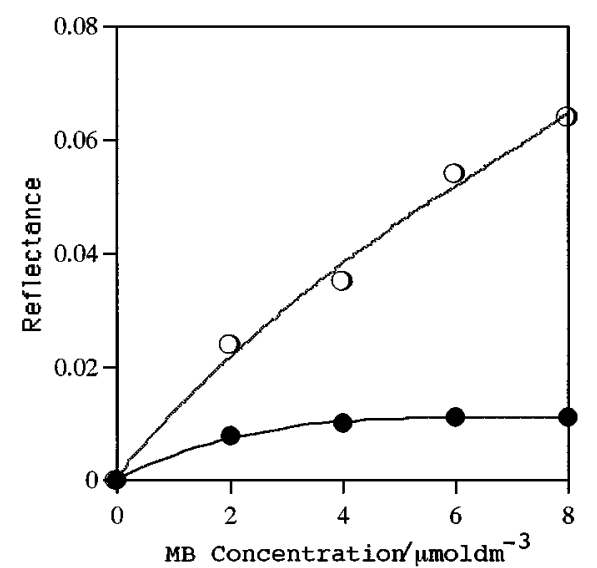

Fig. 8 Response of SPR sensor to Methylene Blue (MB) solution. - MB solution; $O$, MB solution with $0.1 \mathrm{mmol} \mathrm{dm}^{-3}$ dodecylbenzene sulfonate.

3. K. J. Kuhn and L. W. Burgess, Anal. Chem., 65, 1390 (1993).

4. N. Fabricius, G. Gauglitz and J. Ingenhoff, Sens. Actuators B, 7, 672 (1992).

5. W. Lukosz, D. Clerc, P. M. Nellen, C. Stamm and P. Weiss, Biosens. Bioelectron., 6, 215 (1991).

6. N. J. Goddard, D. Pollard-Knight and C. H. Maule, Analyst [London], 119, 583 (1994).

7. E. F. Schipper, A. J. H. Bergevoet, R. P. H. Kooyman and J. Greve, Anal. Chim. Acta, 341, 171 (1997).

8. K. Tsunoda, H. Itabashi and H. Akaiwa, Bull. Chem. Soc. Jpn., 65, 1581 (1992).

9. K. Tsunoda, H. Itabashi and H. Akaiwa, Anal. Chim. Acta, 276, 133 (1993).

10. K. Tsunoda, H. Itabashi and H. Akaiwa, Anal. Chim. Acta, 299, 327 (1994).

11. K. Tsunoda, E. Yamamoto and H. Akaiwa, Chem. Lett., 1996, 919.

12. X. Chen, K. Itoh and M. Murabayashi, Opt. Rev., 3, 351 (1996).

13. K. Tsunoda, H. Ueno and H. Akaiwa, Chem. Lett., 1995, 993.

14. W. Lukosz and C. Stamm, Sens. Actuators A, 25-27, 185 (1991).

15. Ch. Fattinger, H. Koller, D. Schlatter and P. Wehrli, Biosens. Bioelectron., 8, 99 (1993).

16. C. Stamm and W. Lukosz, Sens. Actuators B, 11, 177 (1993).

17. H. Lehmann, M. E. Lippitsch, W. Ecke, W. Haubenreisser, R. Willsch and D. Raabe, Sens. Actuators B, 29, 410 (1995).

18. W. Lukosz, C. Stamm, H. R. Moser, R. Ryf and J. Dubendorfer, Sens. Actuators B, 38-39, 316 (1997).

19. T. Okamoto, J. Spectrosc. Soc. Jpn., 47, 19 (1998).

20. N. J. Harrick, "Internal Reflection Spectroscopy", Harrick Sci. Co., New York, 1987.

21. K. Itoh and M. Madou, J. Appl. Phys., 69, 1 (1991).

22. K. Tsunoda, H. Itabashi and H. Akaiwa, Chem. Lett., 1995, 935.

23. Y. Kano and S. Kawata, J. Spectrosc. Soc. Jpn., 42, 149 (1993). 